\title{
Decision making under
}

\section{uncertainty in a spiking}

neural network model of the

\section{basal ganglia}

Charlotte Héricé ${ }^{1,2}$, Radwa Khali², Marie Moftah ${ }^{3}$, Thomas

Boraud $^{1,2}$, Martin Guthrie ${ }^{1,2}$ and André Garenne $e^{1,2,4}$

${ }^{1}$ Université de Bordeaux, Institut des Maladies Neurodégénératives, UMR 5293, 33000

Bordeaux, France

${ }^{2}$ CNRS, Institut des Maladies Neurodégénératives, UMR 5293, 33000 Bordeaux, France

${ }^{3}$ University of Alexandria, Alexandria, Egypt

${ }^{4}$ andre.garenne@u-bordeaux.fr

Journal of Integrative Neuroscience, Vol. 15, No. 3 (2016) 1-24

Received 26 August 2016; Accepted 23 October 2016; Published 


\section{Abstract}

The mechanisms of decision-making and action selection are generally thought to be under the control of parallel cortico-subcortical loops connecting back to distinct areas of cortex through the basal ganglia and processing motor, cognitive and limbic modalities of decision-making. We have used these properties to develop and extend a connectionist model at a spiking neuron level based on a previous rate model approach. This model is demonstrated on decision-making tasks that have been studied in primates and the electrophysiology interpreted to show that the decision is made in two steps. To model this, we have used two parallel loops, each of which performs decision-making based on interactions between positive and negative feedback pathways. This model is able to perform two-level decision-making as in primates. We show here that, before learning, synaptic noise is sufficient to drive the decision-making process and that, after learning, the decision is based on the choice that has proven most likely to be rewarded. The model is then submitted to lesion tests, reversal learning and extinction protocols. We show that, under these conditions, it behaves in a consistent manner and provides predictions in accordance with observed experimental data.

Keywords: Basal ganglia; decision making; connectionist models; action selection. 


\section{Introduction}

Decision making is a process that permits an organism to choose an action [Lee et al., 2012] from among several alternatives. This process is performed by a complex network consisting of cortical and sub-cortical structures, among which the basal ganglia (BG) and thalamus play a major role. Over the past two decades, the BG have gradually been regarded as involved in selecting motor actions [Mink, 1996, Kropotov and Etlinger, 1999, Redgrave et al., 1999] and are now also known to have limbic and cognitive roles [Bar-Gad et al., 2003]. The structures of the BG therefore have an important role in information processing at the heart of the nervous system. However, even though their patterns of connectivity have been in large part described [Gurney et al., 2001] their complexity is such that they are difficult to analyze formally.

Many models and functional hypotheses have been developed since the original box and arrow description of connectivity was developed more than 25 years ago [Albin et al., 1989, Alexander et al., 1986]. These have attempted to understand the dynamic interactions between the different pathways and their consequences for functionality [Bar-Gad and Bergman, 2001, Mink, 1996, Redgrave et al., 1999, Suri and Schultz, 1999]. For instance in the computational study of Suri [Suri and Schultz, 1999], a single compartment represents a neuronal subpopulation that describes a given region. The transfer of information between the compartments is represented by scalar numeric activity values. This formalism provides mesoscopic views of information exchange and show dynamic characteristics that are compatible with behavioral and electrophysiological experimental results [Berthet et al., 2012, Frank et al., 
2001, Leblois et al., 2006a, Sukumar et al., 2012]. It has the major advantage of being computationally tractable because, in general, a single differential equation allows an entire area to be simulated. Moreover, the parameter space is relatively small generally because the parameters are mainly used to control interactions between compartments. On the other hand, it is infrequent that an attempt is made to describe behavioral findings by means of an integrated neural model. Here we define the integrated neural model as a bottom-up designed architecture in which elementary building blocks represent spiking neurons and synaptic subunits and of which assembly covers a whole neuronal system. For a deeper understanding of complex central nervous system mechanisms, it is necessary at some point to attempt a description at this level. For example, this can be useful to demonstrate the relation between a pathophysiological or pharmacological mechanism described at the cellular scale and its impact on the properties of whole nervous system regions. It is therefore clear that these steps need to be taken in parallel with the development of models at a coarser scale. The idea of an integrated neural model should thus be seen as a supplementary guarantee of validity and an improvement in the predictive capacities of mesoscopic computational models (e.g., population rate-models). Along with the difficulties inherent in neuronal scale models, are the need for a great deal of computational power, the combinatorial explosion of their dimensionality and the resulting difficulty in adjusting the system parameters. Despite these difficulties, some studies of neuronal networks of small and medium size have shown both their feasibility and the pertinence of their results [Sarvestani et al., 2013]. Moreover, large scale projects already exist that apply this approach 
with a view to the integration and multi-scale coherence of the computational model produced [Hines et al., 2008, Migliore et al., 2006].

In this study we have developed a neural network model mainly inspired by previous architecture and dynamical properties initiated by Leblois [Leblois et al., 2006a] and extended by Guthrie [Guthrie et al., 2013]. The initial tasks chosen to test the characteristics of the computational model for this study come from behavioral and electrophysiological recordings made in non-human primates by our team [Piron et al., 2016, Pasquereau et al., 2007]. The neural architecture has been modified [Mink, 1996, Nambu, 2011] to include additional circuitry details. The first difference in our approach is that, instead of using population rate-models, we have implemented spiking neurons and synaptic units circuits with a higher level of detail and dynamics. Moreover we have submitted the resulting model to additional tests and protocols. We have first reproduced previous modeling results regarding the role of BG loops in optimal decision making learning with this new model. We have then shown that it was able to perform classes of tasks that have not previously been demonstrated in this type of model. First the model was able to switch to a different learning task with good performances and without requiring intrinsic modification. Second it was able to perform additional behavioral processes related to such as reversal learning and extinction protocol. Third the model was also able to reproduce the effect of partial lesion studies in a consistent manner. Our spiking neuron-based description of the BG system has thus shown, in addition to several innovative results, its coherence with previous population rate models and moreover, it offers the possibility of investigating 
the effect of cell-level mechanisms on the global BG network properties.

\subsection{Behavioral task}

Electrophysiological and behavioral data have been obtained from macaque monkeys in two center-out reinforcement learning tasks [Pasquereau et al., 2007, Piron et al., 2016]. In the first task, a typical session consisted of series of trials in which four different target shapes (four-cue protocol) were used, each associated with a different reward probability $(\mathrm{P}(\mathrm{R}))$ of $0,0.33,0.66$ and 1$)$. On each trial, two of the cues were selected pseudo-randomly such that each of the six possible cue combinations was presented 20 times in a complete session. Our simulation sessions thus contained 120 trials. In the second task only two different cues were used with $\mathrm{P}(\mathrm{R}) \mathrm{s}$ of 0.25 and 0.75 . In both cases, the aim for the monkeys was to select the cue with the highest reward probability in order to maximize the reward delivered (fruit juice). Extra-cellular recordings were performed simultaneously in the left Globus Pallidus pars interna (GPi) and in the dorsal striatum.

Analysis of the electrophysiology suggests that the cues used in the first behavioral task [Pasquereau et al., 2007] can be decomposed into a cognitive aspect (the cue shape) and a motor aspect (the direction to move). We have therefore modeled this as two sets of action selection loops in parallel. Each action selection loop is represented as an area of cortex in closed-loop feedback through the BG with itself. These loops also comprise associative areas of cortex and striatum that represent a compression of the two dimensions of the cue. Associative cortex inputs to the striatum are assumed to be a high-level 
visual representation (arising from parietal cortical areas) that is a combination of both the cue shape and position [Guthrie et al., 2013]. The four possible cue shapes are associated with four cognitive cortical subpopulations and a similar distribution is applied to direction/motor subpopulations. There are therefore 16 possible combinations of cue shape and direction, each represented by a subpopulation in associative cortex and striatum. For instance if two cues are shown in two positions on each trial, only two of the cognitive, two of the motor and two of the corresponding cortical associative subpopulations will be activated on each trial. For clarity and ease, we will use the following nomenclature in this paper. The target shapes will be named $\mathrm{C} 0, \mathrm{C} 1, \mathrm{C} 2$ and C3 (where C means cortical "cognitive" subpopulations) and the motor directions will be named M0, M1, M2 and M3. The P(R) will be distributed as follow: C0: 0, C1: 0.33, C2: 0.66 and $\mathrm{C} 3$ : 1 . The second task has two different cues with $\mathrm{P}(\mathrm{R})$ of 0.25 and 0.75 that are represented in the same four locations and processed in a similar manner (two-cue protocol).

\subsection{Model architecture}

A general schema of the circuitry involving the cortex, BG and thalamus is shown in Figure 1. There are three main pathways that form cortico-cortical loops through the BG. The direct pathway (i) overall exerts an excitatory (positive) feedback that is convergent and focused [Percheron et al., 1984]. In contrast, the hyperdirect pathway (ii) exercises an inhibitory feedback that is widespread due to divergence of connections from the subthalamic nucleus (STN) to the GPi. The interaction between these two pathways has a tendency 
to amplify any difference between cortical networks activating different direct pathway loops [Mink, 1996]. The indirect pathway (iii) has not been included in this modeling study for various reasons. It was originally thought to provide a closed, negative feedback loop [Albin et al., 1989], but with further delineation of anatomical connections, would seem to form a sub-network with internal positive and negative feedback loops, at the heart of the BG [Bevan et al., 1997, Kita and Kita, 1994, Smith and Bolam, 1989]. With this proliferation of anatomical complexity, it is no longer clear that the indirect pathway forms a closed cortico-subcortical loop that would be able to transfer precise spatiotemporal information [Levy, 1997]. Divergence both in the Globus Pallidus pars externa (GPe)-STN and the GPe-striatum sub-loops [Bevan et al., 1997, Chang et al., 1981, Gerfen, 1985] as well as local GPe axon collaterals [Bevan et al., 1997, Brown et al., 1998] would suggest that this is unlikely.

We have also taken into account the effect of medium spiny neurons lateral inhibition (LI) in striatal populations of our model. The exact functional role of these connections remains mostly unknown or controversial but in a neuronal population LI often plays a role in information processing [Schaette and Kempter, 2012, Ghodratitoostani et al., 2016]. Recent studies has emphasized LI involvement in the striatum where it tends to increase the signal-to-noise ratio [Moyer et al., 2014] and also the BG capability to deal with complex tasks [Berthet et al., 2016].

The network inputs are sensory representations of the environmental cues and the action selection occurs over the course of many circuits through the loops. 


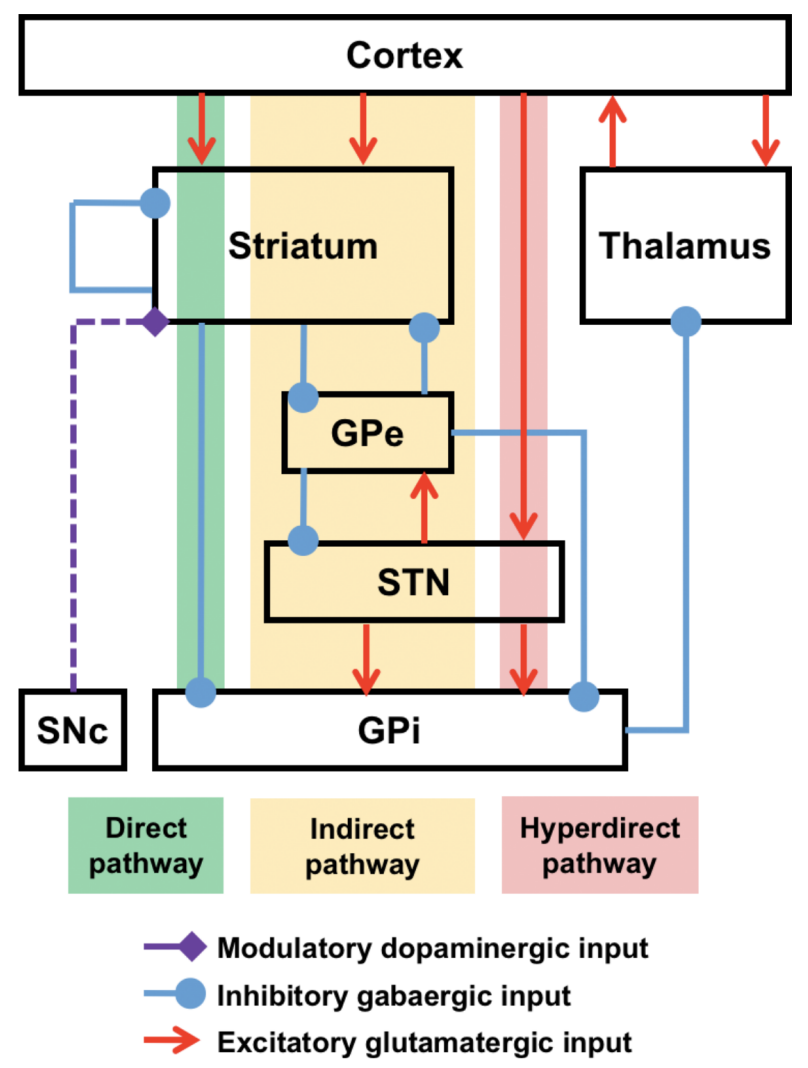

Figure 1: Box and arrow diagram of the cortico-basal ganglia network and pathways, based on [Bar-Gad et al., 2003, Moyer et al., 2014].

The model architecture is initially based on that of Leblois [Leblois et al., 2006a] and Guthrie [Guthrie et al., 2013] and has been adapted to represent the task under consideration and additional circuitry (cf. Figure 2). The small number of neurons in each subpopulation is a tradeoff which approximately takes into account relative structure sizes [Schroll et al., 2014]. It was chosen for computational tractability and not to represent exact neuronal population ratios [Humphries et al., 2006]. The subpopulation sizes are: cortex, 100; striatum, 40; STN, 10; GPi 10; thalamus, 20. The total network size is 3,680 
neurons and the connectivity ensemble between regions comprises 723,200 synapses. This provides a network of sufficient size to produce realistically complex synaptic interactions, but still computationally tractable on a standard desktop computer.

\subsection{Analysis of cortical activity}

As well as representing the salience of the sensory inputs, cognitive and motor cortical activity also serve as detectors of the choice made and therefore the behavior of the animal. Presentation of the cues is simulated by an additional current injection into the corresponding cortical subpopulations [cf. Figs. 3(b) and 3(c)]. Each simulated trial lasts 1 second from the presentation of the stimuli. Each ms, the spikes timestamps of each neuron are recorded in parallel with behavioral events, reward scores and cognitive cortico-striatal synaptic weight values. For each neuronal subpopulation i of size $\mathrm{N}_{\text {neurons }}(\mathrm{i})$, an average firing rate (i) is computed each ms and expressed in $\mathrm{Hz}$ :

$$
\alpha(i)=\frac{N_{\text {spikes }}(i)}{N_{\text {neurons }}(i)} \cdot 10^{3},
$$

where $\mathrm{N}_{\text {spikes }}(\mathrm{i})$ is the number of recorded spikes during this ms. To link the spiking activity of the model to the behavior and thus to define when the decision to choose a particular target is made, we compute the difference in average firing rates between the two activated subpopulations. When this difference surpasses a threshold value of $40 \mathrm{~Hz}$ [Guthrie et al., 2013] this is referred to as symmetry breaking and the movement selection is then designated 


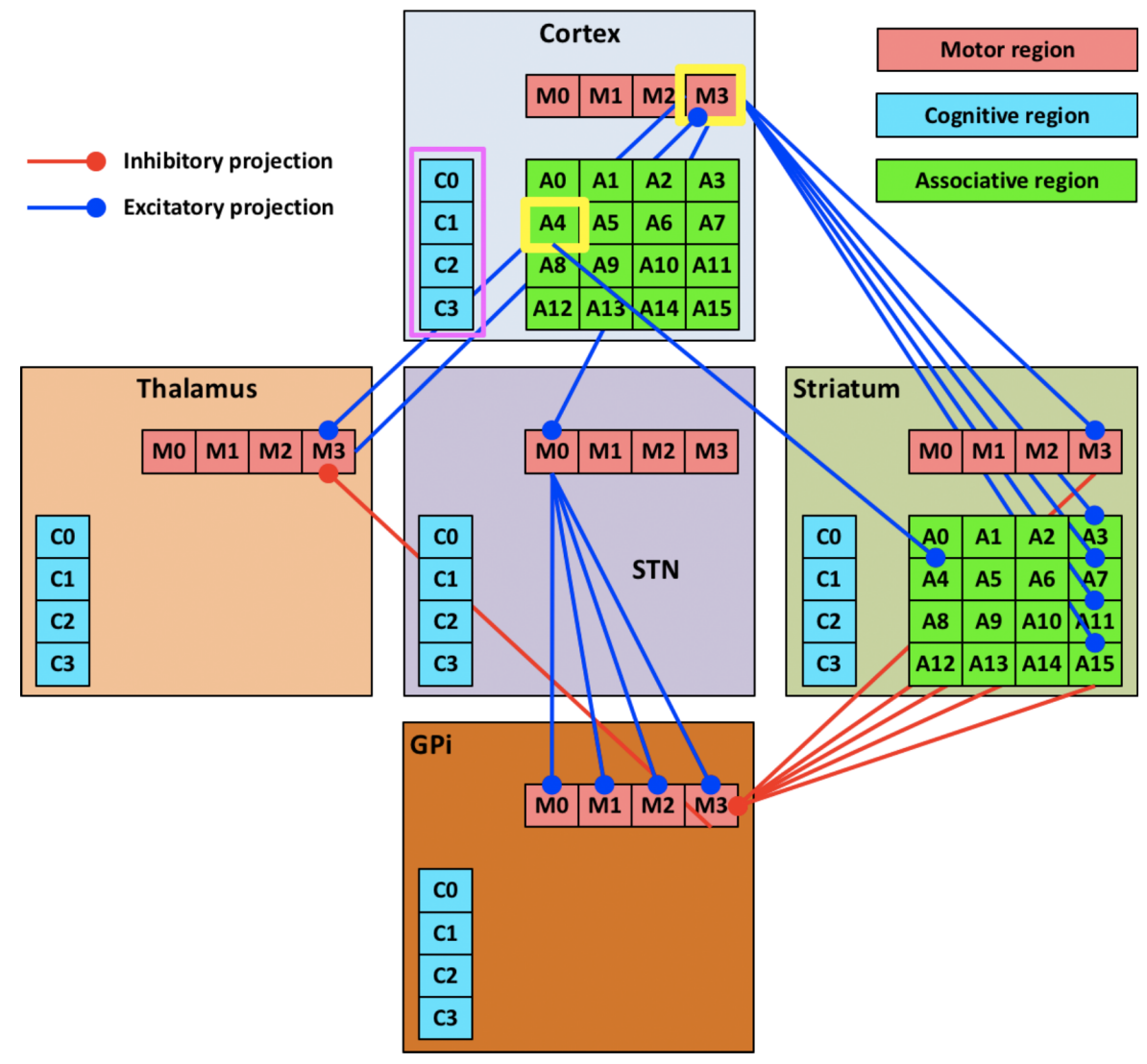

Figure 2: (Color online) Detailed architecture of the 72 regions of the model. The cortico-basal ganglia loops are described taking into account the two levels of decision making: (i) a cognitive loop (blue subpopulations) and (ii) a motor loop (pale red subpopulations). The cortical and striatal associative regions are in green. Each cell of the grid (for instance $M 1$ or C3) represents a neuronal subpopulation which size depends on the region. A partial connectivity template taking into account M3 and A4 cortical inputs is shown here (for the sake of clarity the complete connectivity pattern is not shown). The plastic synapses considered in the model exclusively arise from the cognitive (magenta frame) cortico-striatal projections. The central part of the figure represents the hyper-direct pathway (cortex - STN - GPi) whereas the right side represents the direct pathway (cortex - striatum - GPi).

by the cortical motor subpopulation that has the highest firing rate. During a trial this symmetry breaking can be seen in both the cognitive and motor loops. In the motor cortex, it is interpreted as the onset of a movement in one 
direction (M0, M1, M2 or M3). A reward is then given or withheld based on the $\mathrm{P}(\mathrm{R})$ of the target chosen by the movement. If the movement is toward the target shape with the highest $\mathrm{P}(\mathrm{R})$, this is considered as a good choice (GC) even if no reward is given. Similarly a good decision (GD) occurs when the activity of the cognitive cortical subpopulation associated with the highest $\mathrm{P}(\mathrm{R})$ is greater than the activity of the cognitive cortical subpopulation associated with the other presented cue.

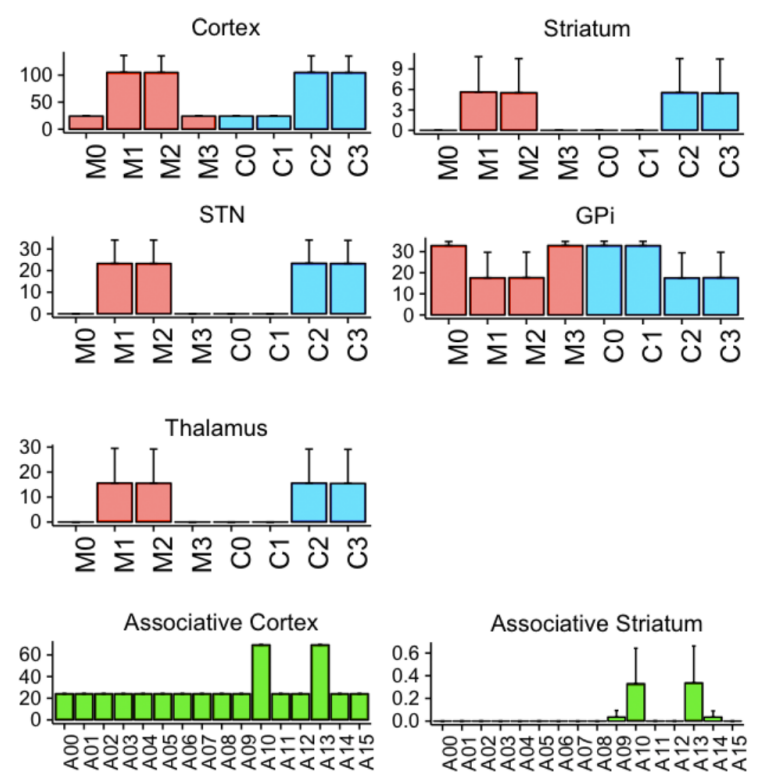

(a)

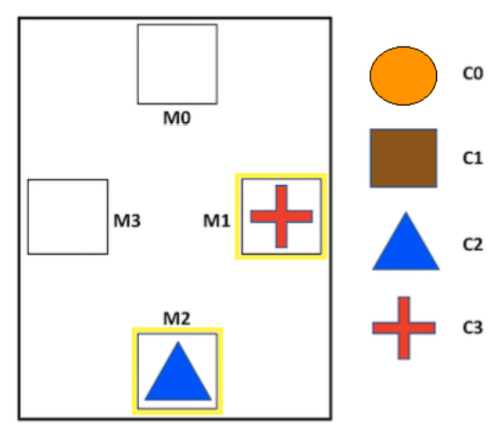

(b)

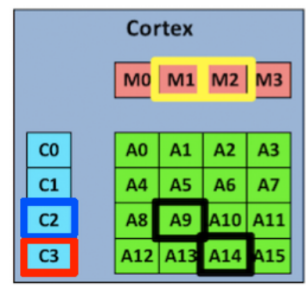

(c)

Figure 3: (Color online) (a) Average neuronal firing rates (Hz) pooled from 30 different networks recorded over 30 trials of 1 s each $(N=30 ; p<0: 05$; one wayANOVA). Error bars represent standard deviation of the pooled data. (b) Combination sample of displayed targets during the task with their shape/color and localization on the screen and (c) encoding of the target presentation in the corresponding cortical subpopulations (yellow for motor, black for associative and dark blue and red for the two cognitive subregions). 


\subsection{Model implementation}

This study required a neuronal model that was sufficiently simple to be implemented in a large-scale network. To restrict the complexity and the size of the parameter space of the model, we have chosen a leaky integrate-and-fire neuron (LIF):

$$
\begin{gathered}
C \cdot \frac{d V_{m}(t)}{d t}+\frac{V_{m}(t)-V_{\text {rest }}}{R}=I(t)+\xi(t), \\
\quad \text { if } V_{m}(t)>V_{\text {thres }}: V_{m}(t) \leftarrow V_{\text {rest }},
\end{gathered}
$$

where $V_{m}(t)$ is the membrane potential and $\mathrm{V}_{\text {rest }}$ the resting potential. Default values are: $\mathrm{C}=10^{-10} \mathrm{~F}, R=10^{8}$ ohms, $V_{\text {rest }}=-65 \mathrm{mV}, V_{\text {thres }}=-55 \mathrm{mV}$ and refractory period is $1 \mathrm{~ms}$. $\xi(\mathrm{t})$ is a gaussian white noise current which simulates synaptic bombardment [Neymotin et al., 2011] and $I(t)$ the externally applied current. The network was built using voltage-jump weighted synapses. The interneuronal transmission delays based on Humphries et al., (2006) and weights necessary for coherent model dynamics are summarized in Table 1. To simulate the network physiological basal activity, a base current was applied in the network in addition to the synaptic noise $\xi(\mathrm{t})$.

The model was developed with GNU C++ 4.9 library and numerical equations were solved using a first order forward Euler algorithm with a one ms time step. Simulation were run using Monte Carlo methods. Data analysis and visualization were performed with python-matplotlib [Hunter, 2007] and R [R-Team, 2015]. 
Table 1: Synaptic weights and delays used in the connections between subpopulations of the circuit (M: motor, C: cognitive, A: associative).

\begin{tabular}{|c|c|c|c|c|}
\hline \multicolumn{3}{|c|}{ Connexion } & \multirow{2}{*}{$\frac{\text { Weight (mVolt) }}{0.1}$} & \multirow{2}{*}{$\frac{\text { Delay }(\mathbf{m s})}{11}$} \\
\hline Cortex (M/C) & $\rightarrow$ & Striatum $(\mathrm{M} / \mathrm{C})$ & & \\
\hline Cortex (M/C) & $\rightarrow$ & Striatum (A) & 0.035 & 11 \\
\hline Cortex (A) & $\rightarrow$ & Striatum (A) & 0.02 & 11 \\
\hline Cortex (M/C) & $\rightarrow$ & STN & 0.125 & 6 \\
\hline Cortex $(\mathrm{M} / \mathrm{C})$ & $\rightarrow$ & Thalamus & 0.125 & 5 \\
\hline STN & $\rightarrow$ & GPi & 2 & 2 \\
\hline Thalamus & $\rightarrow$ & Cortex & 10 & 5 \\
\hline Striatum & $\rightarrow$ & GPi & -5 & 6 \\
\hline Striatum & $\rightarrow$ & Striatum & -0.25 & 2 \\
\hline GPi & $\rightarrow$ & Thalamus & -10 & 5 \\
\hline
\end{tabular}

\subsection{Plasticity and learning}

As this first study is essentially looking to show how the decision making capabilities and learning take place, we have chosen to implement a simple LTP/LTD weight update rule driven by the presence or absence of a reward following a move in an outcome specific manner [Cools et al., 2009]. In the model, learning occurs only at the cognitive cortico-striatal synapse where phasic changes in dopamine concentration have been shown to be necessary for the production of long-term synaptic weight variations [Pawlak and Kerr, 2008, Kerr and Wickens, 2001, Reynolds et al., 2001]. When a chosen target results in a reward, all the weights of the cognitive cortico-striatal projections contributing to the choice of this target are slightly increased. In all other outcome situations, they are decreased. The synaptic weight variation rule is computed as follow: 


$$
w_{i, j}(n+1)=w_{i, j}(n)+\eta \cdot \Delta G, \quad \eta=\frac{w_{\max }-w_{i, j}(n)}{w_{\max }-w_{\min }}
$$

where $w_{i, j}$ is the weight of the synapse between cells $i$ and $j, \eta$ the learning rate and $\Delta G$ an increment that was set to $5 \%$ of the average weight values. $\eta$ depends on the current $w_{i, j}$ value and the resulting weight variation curve follows a Sutton-Barto model [Klopf, 1988].

\section{Results}

\subsection{Parameterization and model behavior}

The first stage of the setup of the model was to simultaneously adjust the various parameters to produce average firing rate activities in the different regions that were in accordance with those observed in vivo. For example, striatal activity is generally very low compared to cortical activity or GPi activity [Pasquereau et al., 2007]. In the same way, thalamic activity shows large variation in the model, but the range of frequencies observed remains in accordance with experimental data [Chen et al., 2010].

As previously mentioned, we apply a Monte Carlo approach to run our simulations. Due to randomization in the assignment of initial weights and conduction delays, each network model is unique. To rule out the possibility of a sample effect on the functional properties of the networks, 30 simulations were run for each of the six possible cue combinations. Figure 3(b) shows a combination composed of M2-C2 for target 1 (blue triangle) and M1-C3 for target 2 (red 
cross). This configuration is encoded in the model by the stimulation of the corresponding framed subpopulations [cf. Fig. 3(c)]. The firing rates recorded in the various cognitive, associative and motor subpopulations remains globally consistent with the input pattern and no significant difference was found in each of the 72 subregions between the 30 generated networks. Therefore, there is no noticeable "network effect" on the model dynamics and all of the generated models behave in a comparable way.

As shown in Fig. 3(a), the stimulated cortical regions have a higher average frequency, as would be expected. Referring to Fig. 2 for the connectivity, propagation of information through the network can also be observed. The striatal cognitive and motor subpopulations are activated in a similar pattern to the corresponding cortical areas. Subpopulations in the associative striatal region receive the sum of cognitive, motor and associative cortical inputs. In Figure 3(a), peaks in A10 and A13 and, to a lesser extent (due to the lack of additional associative cortex input) in A9 and A14 can be observed in striatal subpopulations. Activity in the STN is aligned with that of cortex. In the GPi the activities observed are the inverse of those seen in the cognitive and motor subpopulations of striatum. This is consistent with the known action of the inhibitory outputs from striatum. Finally, thalamic activity is balanced by excitation from cortex and inhibition from GPi and differences in activity are therefore amplified. This amplification is the mechanism that leads to symmetry breaking, where the activity in one channel increases while that in the other channel decreases. 


\subsection{Dynamic action selection}

In the absence of learning the network is still able to make a decision. At the start of a trial, the presentation of the two targets leads to the activation of two channels in the positive feedback direct pathway loop. Symmetry breaking between the two channels occurs as follows: because the loop gain of the direct pathway is greater than one, differential activation of one channel due to noise is amplified. The resultant increased cortical activation starts a positive feedback takeoff in that channel specifically because direct pathway channels are segregated. The increase of average firing rates measured in this channel finally reaches a plateau that leads to a stabilization of all the subregions activities in the system [cf. Fig. 4(a)]. This upper bound results from the model constraints, notably regarding the circuitry, the conduction delays and the cellular properties. But the increased cortical activation of one channel also increases the input to the divergent, negative feedback hyperdirect pathway causing suppression of all channels, especially the competing channel. A small difference in channel activations due to noise thus leads to one channel being highly activated to the detriment of the others.

In a naïve network, the symmetry breaking should select one of the two subpopulations with equal probabilities and the average GC rate should be close to 0.5 . Similarly, the average reward rate should correspond to the average probability value: $(1+0.66+0.33+0) / 4=0.5$. As shown in Figure $4(\mathrm{~b})$, our simulations results are consistent with these predictions and in the absence of learning, the GC and reward rates are both close to 0.5. 

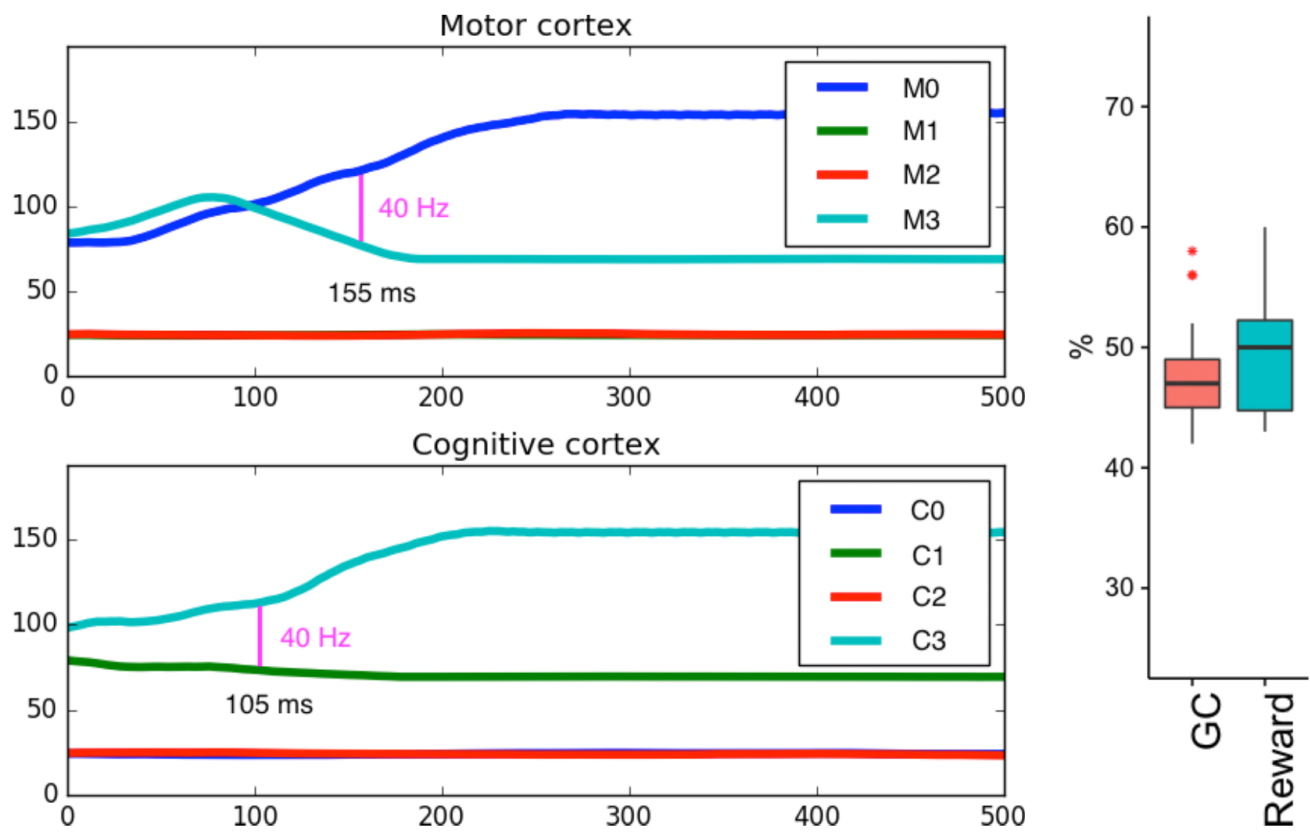

(a)

(b)

Figure 4: (a) Recording of the average firing rate in the subpopulations M0, M3, C1 and $C 3$ following the simultaneous presentation of the target $C 3$ at the MO location and of the target $C 1$ at the $M 3$ location. This trial illustrates a GC example since the selected motor activity is $M O$ which corresponds to the direction of the presentation of the shape $C 3$ which has the highest $P(R)$. (b) Average percentage of reward and $G C$ rates in 16 sessions of 100 trials. Both scores are consistent with $50 \%$ chance $(N=16 ; p<0: 05 ;$ two-sided Wilcoxon test).

The symmetry breaking events onsets are effective when a $40 \mathrm{~Hz}$ average firing rate difference is detected between two subpopulations. Figure 4 shows an example of a decision making (cognitive level) preceding an action selection (motor level) by $50 \mathrm{~ms}$. For practical reasons symmetry breaking delays are computed from the smoothed firing rate curves difference. A 100 ms sliding window averaging convolution was applied. Changing the window size only slightly shifts the action selection times but the relative delays between cognitive and motor threshold onsets as well as the event orders are not affected. 


\subsection{Sensitivity study}

\subsubsection{Effect of noise and base current on symmetry breaking in the model}

Both noise and base current amplitude [cf. (refsec:implementation)] affect the network ability to generate symmetry breaking. As shown in Fig. 5(a), setting noise amplitude too low or too high decreases its symmetry breaking score in the motor cortex (i.e, its action selection capability) and the optimum noise level of our model is close to $50 \mathrm{pA}$.

When the base current is too low, there is not enough gain in the BG direct pathway loops to destabilize their relative activities and no salience takes place between the motor cortex subpopulations. When it is too high, it tends to exceed its external inputs and therefore to drive the whole network activity in a balanced way which prevents symmetry breaking [Fig. 5(b)]. As with the noise level, there appears to be an optimum value to apply which is close to $100 \mathrm{pA}$.

We have then chosen these intermediate values of $50 \mathrm{pA}$ for the noise and $100 \mathrm{pA}$ for the base current in order (i) to maximize the network symmetry breaking performances but also (ii) to provide average firing rates consistent with those recorded in the different regions in vivo. 


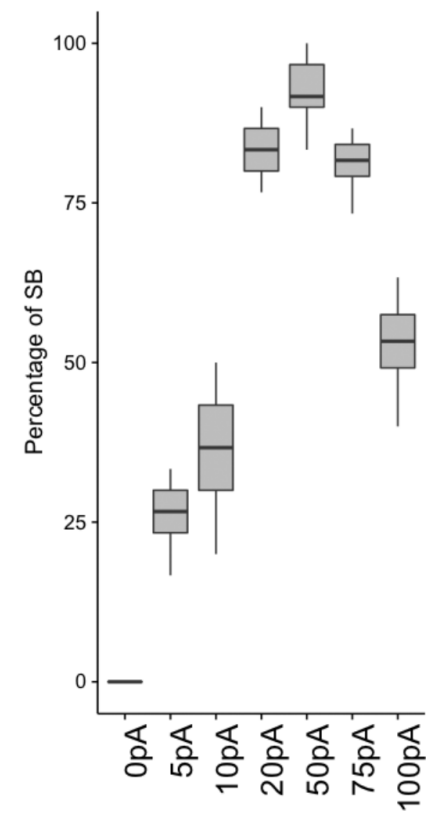

(a)

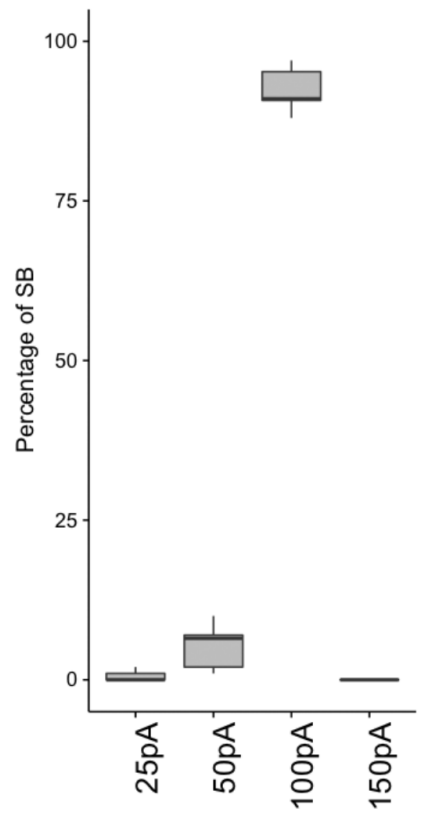

(b)

Figure 5: Effect of $\xi(t)$ (current noise amplitude) and (b) basal input current on the percentage of symmetry breaking in the motor cortex (SB) following the presentation of two random targets at two different positions $(N=16 ; p<0: 05$, Kruskal-Nemenyi multiple comparison test).

\subsubsection{Lesion studies}

Simulations have been performed to test the impact of lesions at each point in the circuit on network regions activity. 30 sessions were run on 8 different networks in four conditions. For each condition a given interconnection was selectively interrupted (synaptic weights set to 0) and the activities of different regions were recorded, pooled and averaged. The conditions used were control (intact) model, striato-pallidal lesion, cortico-thalamic lesion and pallido-thalamic lesion. The results are summarized in Fig. 6.

The striato-pallidal (direct pathway) lesion initially increases the GPi activity 
but rapidly, this increase inhibits in turn the thalamus and therefore the cortical sub-regions. Since the model activity is mainly driven by the cortex, the net effect of this lesion is then a global decrease in all subregions average firing rates.

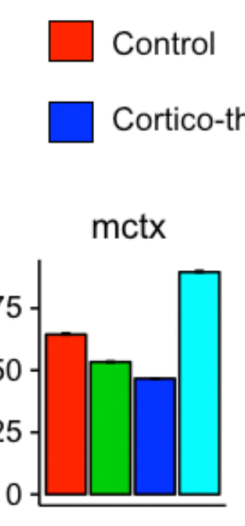

mstn

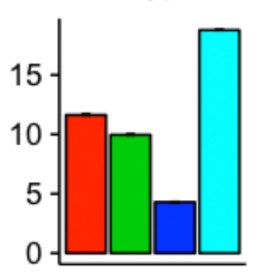

mtha

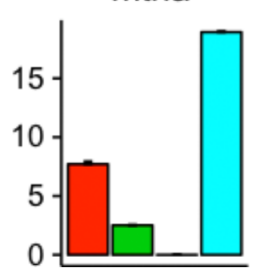

$\operatorname{cct} x$
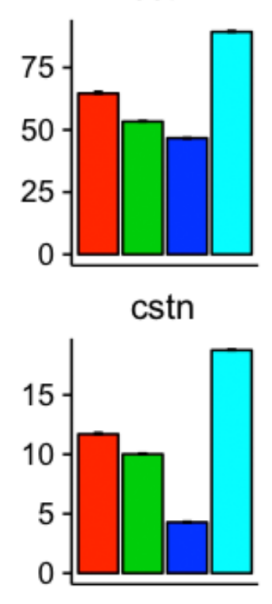

ctha

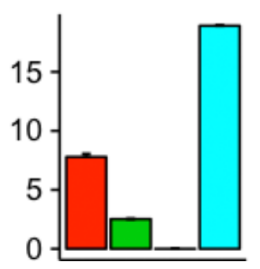

Striato-pallidal lesion

Pallido-thalamic lesion

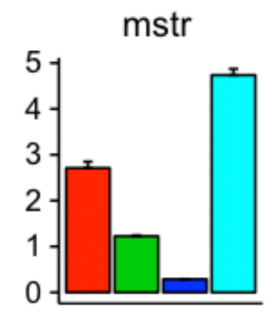

mgpi

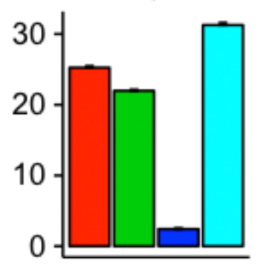

actx

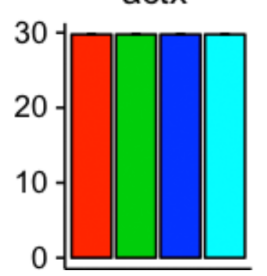

cstr

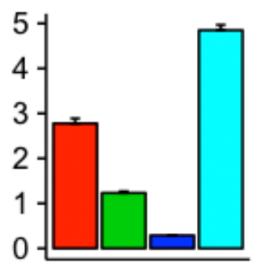

cgpi

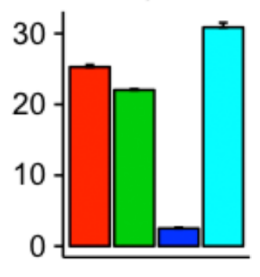

astr

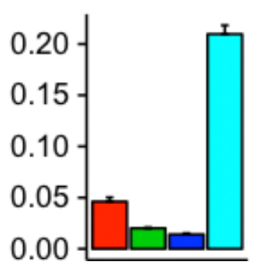

Figure 6: Effect of selective lesions on the network populations average firing rates $(\mathrm{Hz})$ recorded in 8 different networks over 30 sessions. $c_{X}, m_{X}$ and $a_{X}$ : respectively cognitive, motor and associative $X$ population. The populations abbreviations are: ctx for the cortex, str for the striatum, stn for the sub-thalamic nucleus, gpi for the GPi and tha for the thalamus.

The cortico-thalamic lesion also generates a global attenuation of firing rates. 
Since the cortical inputs are the main driving force of the network activity and since the cortico-thalamic pathway is a positive gain feedback loop, when this connection is removed, the only remaining input to the thalamus is the inhibitory GPi input. The spontaneous thalamic activity is then drastically reduced and consequently the cortical activity. Therefore, as with the striatopallidal lesion, the global network firing rate decreases.

The pallido-thalamic lesion is also a consistent qualitative control of the behavior of our model. When the inhibitory projections from the GPi to the thalamus are removed, the brake that was exerted on the thalamo-cortical structures is stopped. The mutual interaction between the thalamus and the cortex becomes then more efficient which leads to an increase in the cortical regions firing rates and therefore in all the network subpopulations.

\subsection{Learning properties}

\subsubsection{The optimum cue shape direction was preferentially selected}

During training our model learns to create a dynamic link between the cognitive and motor sensory components of a cue without having to resort to rewiring cortical motor projections. This can be seen from the profile of the learning curves of the model. Indeed, the average reward and GC rates gradually increase over the course of the session as shown in Fig. 7(d). As a result, both the GCs and the GDs are significantly improved during a standard learning session of the four-cue protocol [Fig. 7(a)]. The two-cue protocol produces similar results [Fig. 7(b)] and achieves an even higher success rate for GCs and GDs. This better performance can be explained by the larger difference in the 
respective $\mathrm{P}(\mathrm{R})$ of the two targets and by the resulting decrease in uncertainty.

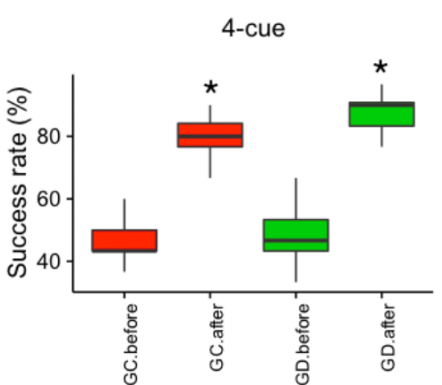

(a)

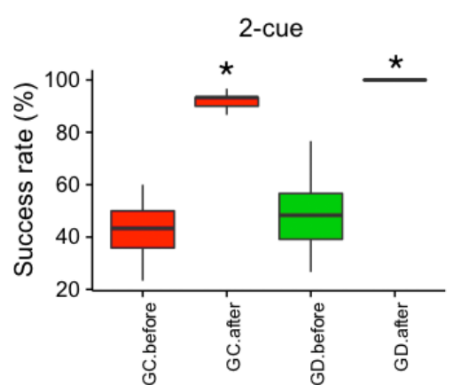

(b)

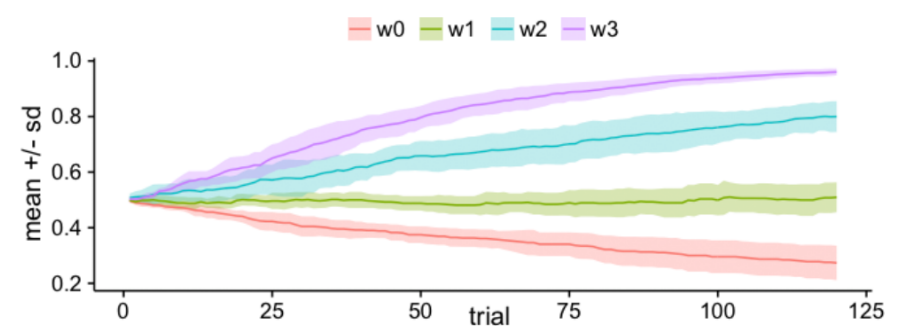

(c)

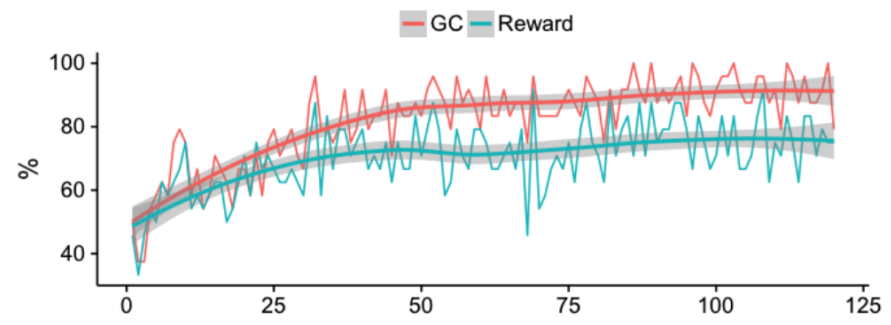

(d)

Figure 7: Effect of learning on model behavioral metrics in (a) four-cue protocol and (b) two-cue protocol, "before" refers to the first 30 trials of a session and "after" to the last 30 trials $(N=16 ; p<0.05 ;$ Wilcoxon test $)$. (c) change in average cognitive cortico-striatal synaptic weights during a training session. $w_{i}$ is the average weight for cue $_{i}$. In these simulations, the higher the index number (i), the higher the associated cue reward probability $\left(P_{i=0}(R)=0, P_{i=1}(R)=0.33, P_{i=2}(R)=0.66, P_{i=3}(R)=1\right)$. (d) Normalized rate of $G C$ and rewards averaged over 24 sessions of the four-cue protocol. The smoothed curves are obtained by a local polynomial regression fitting combined with a $95 \%$ confidence interval. 
The greater the cognitive cortico-striatal weight, the higher the gain in that channel. This leads the channel with the higher cortico-striatal synaptic weights being consistently selected [Fig. 7(c)]. Simultaneously the hyperdirect pathways excite GPi regions, which selectively inhibit the thalamic inputs projecting to the cortical regions whose striatal projections have the lowest weights. Cutting the thalamo-cortical projections in the circuit stopped the symmetry breaking (data not shown) showing that it was due to the action selection properties of the network and not to the increased salience of one target.

\subsubsection{The movement onset delay decreases with learning}

The movement onset (which can be equated to reaction time) is defined as the time between the appearance of the two cues and the symmetry breaking in cortical motor subpopulations. With learning, the time for movement onset decreased (Figs. 8(a) and 8(b)]. This is in accordance with previous modeling studies [Guthrie et al., 2013] as well as with experimental results [Piron et al., 2016].

\subsubsection{Striatal LI plays an important role in model learning capability}

The absence of LI (NLI condition) impairs both levels of the decision making process [Figs. 8(c) and 8(e)]. This tends to confirm its role in gain control [Moyer et al., 2014].

NLI decreases the GC rate in a naïve network [Fig. 8(c)-left] and abolishes its learning capability (Fig. 8(c)-right]. This latter result seems related to the more general fall of the symmetry breaking score shown in Fig. 8(d). NLI condition 


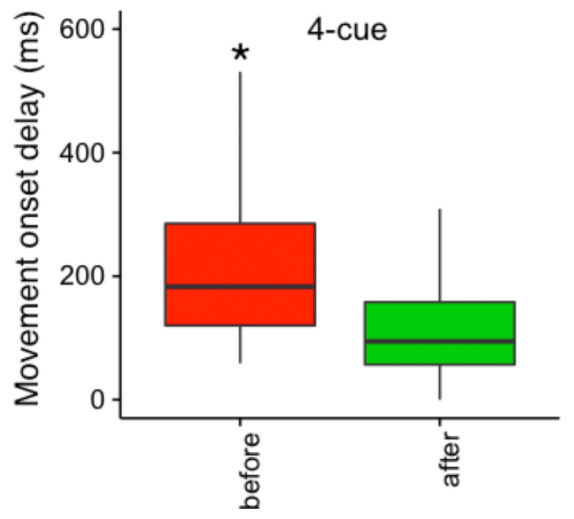

(a)

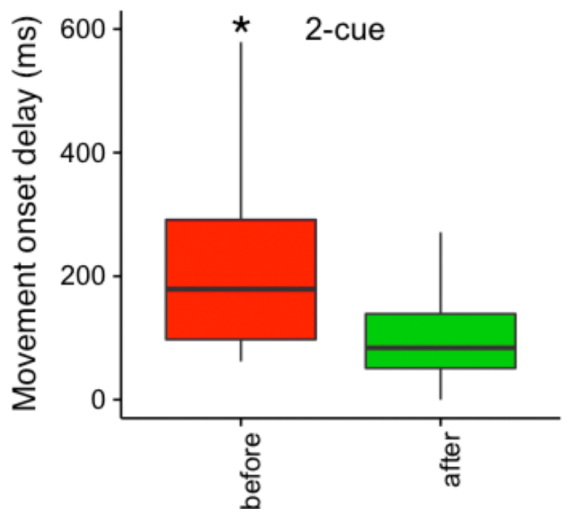

(b)

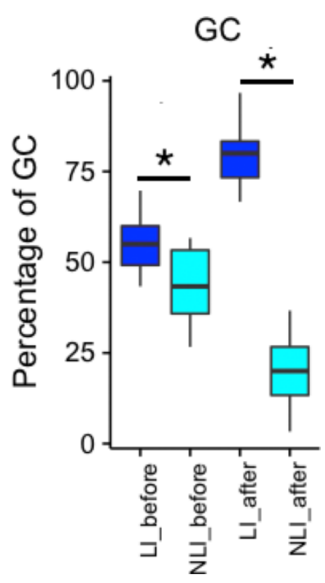

(c)

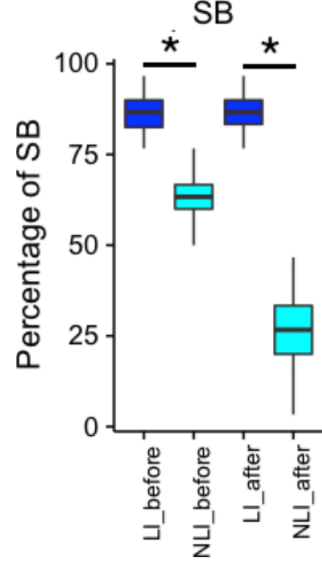

(d)

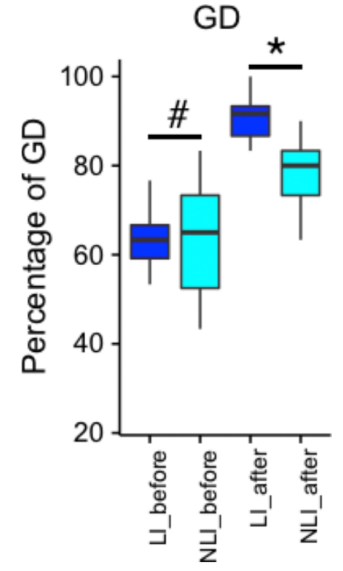

(e)

Figure 8: Top: average movement onset delay is decreased by learning in (a) fourcue protocol and (b) two-cue protocol, "before" refers to the first 30 trials of a session and "after" to the last 30 trials $(N=16 ; p<0: 05$; Wilcoxon test), Bottom: effect of striatal LI (or its absence NLI) on learning performances of the model (c) GC score significantly differs both before and after learning when LI is absent (d) the NLI condition dramatically decreases the number of SB motor events. This indicates that a motor selection occurs less frequently both before and after learning (e) after learning GD score depends on $L I(N=16 ; p<0: 05$; Wilcoxon test $)$.

does not impact the GD score in a naïve network [Fig. 8(e)-left] but after training it tends to decrease the final GD score [Fig. 8(e)-right]. This effect can be partially attributed to a deficient action selection process that prevents 
the model from reaching its optimum decision making performance. Together, these findings show a possible role of LI in striatal information processing and consequently, in the learning process.

\subsection{Reversal learning protocol}

Reversal learning consists of an $n$-step training procedure where the subject has to adapt its behavior to gain reward after a contingency change. This method is often used to unravel neuropsychological mechanisms [Dombrovski et al., 2015, Cools et al., 2002, Costa et al., 2015]. To assess our model learning characteristics, we have implemented a reversal learning protocol which is summarized in Table 2.

Table 2: Reversal protocol description.

\begin{tabular}{rllll}
\hline Cue number & C0 & C1 & C2 & C3 \\
\hline Step 1 (120 trials) & $\mathrm{P}(\mathrm{R})=0$ & $\mathrm{P}(\mathrm{R})=1 / 3$ & $\mathrm{P}(\mathrm{R})=2 / 3$ & $\mathrm{P}(\mathrm{R})=1$ \\
Step 2 (240 trials) & $\mathrm{P}(\mathrm{R})=2 / 3$ & $\mathrm{P}(\mathrm{R})=1$ & $\mathrm{P}(\mathrm{R})=0$ & $\mathrm{P}(\mathrm{R})=1 / 3$ \\
Step 3 (240 trials) & $\mathrm{P}(\mathrm{R})=0$ & $\mathrm{P}(\mathrm{R})=1 / 3$ & $\mathrm{P}(\mathrm{R})=2 / 3$ & $\mathrm{P}(\mathrm{R})=1$ \\
\hline
\end{tabular}

In step 2, the reward contingencies were changed and their new distribution was chosen to maintain an homogeneous $\mathrm{P}(\mathrm{R})$ difference for all cues of $2 / 3$. The model behavior is very similar to what has been observed experimentally [Xue et al., 2013, Morita and Kawaguchi, 2015]. Each new step was followed by an adaptation of the model that successfully improved its score after several tens of trials [Figs. 9(a)-9(c)]. However, each new step is followed by a short 
period of time during which GC score drops down below its initial average $50 \%$ rate. This can be explained by the fact that, during these periods, the model spontaneously selects what it considers as a GC but with inverted $\mathrm{P}(\mathrm{R})$ and thus, it temporarily decreases its reward rate and therefore its learning rate. This process can also be visualized through the cortico-striatal cognitive projection strength evolution [Fig. 9(b)]. In summary it takes more trials to reach an optimum GC rate after each reversal learning step.

\subsection{Extinction protocol}

The extinction phenomenon is a common feature of most of the associative learning processes [Barker et al., 2014]. We have applied to the model a simple response extinction protocol [Goodman and Packard, 2015, Papachristou et al., 2013] which comprised the same training procedure as before, followed by the cessation of reward during 120 additional trials.

We observed that the GC performance gradually decreased [Fig. 10(a)] and exhibited statistically significant differences before and after extinction [Fig. 10(a)- inset]. This occurred in parallel with the cortico-striatal weights variation over time [Fig. 10(b)]. The GC profile is consistent with the general extinction process as described in classical conditioning context. Indeed when it is deprived from reward, the model spontaneously loses its capacity to optimize its choices and gradually "forgot" what it learnt.

We also tested the model behavior when a recall was applied. Experimentally, the conditional response to a cue recall following a learning extinction is faster 


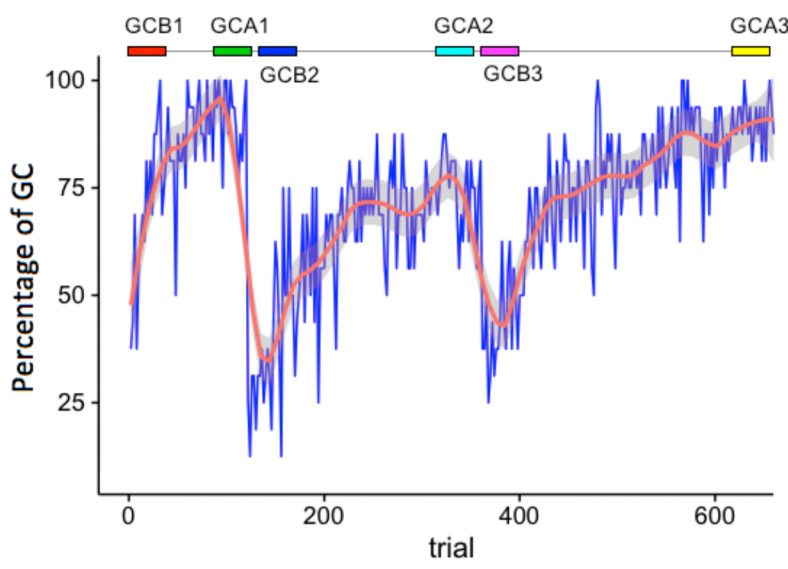

(a)

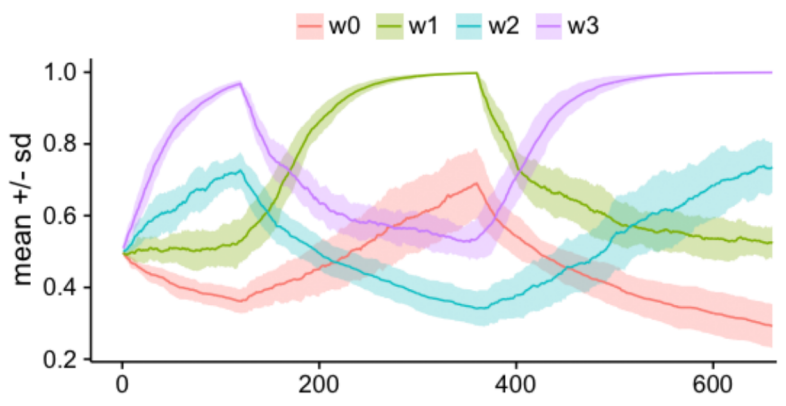

(b)

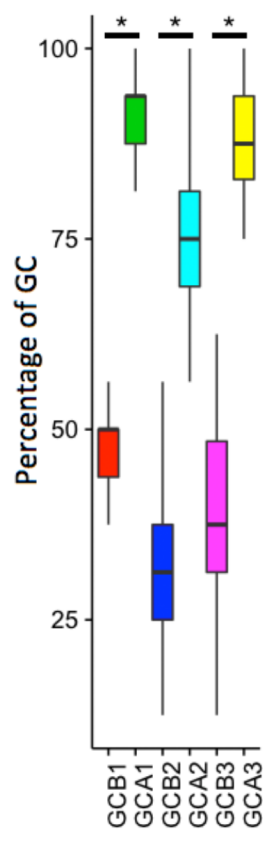

(c)

Figure 9: (Color online) 3-step reversal learning protocol simulation results (a) Normalized rates of GC averaged on 16 sessions (red: smoothed curve obtained by a local polynomial regression fitting combined with a 95\% confidence interval). To compare the efficiency of reversal learning 30 trials samples (color boxes) were analyzed at the beginning and at the end of the three steps (GCBi: good choice before step $i$ and GCAi: good choice rate after step i) (b) variation of average cortico-striatal cognitive synaptic weights over a training session. $w_{i}$ is the average weight value for each of the four cues. (c) GC rate comparison before and after training at each step ( $N=16 ; p<0: 05$, Kruskal-Nemenyi multiple comparison test).

than the in the initial condition [Bouton, 2004] and this phenomenon is even observed in invertebrates [Sandoz and Pham-Delegue, 2004]. As shown in Figs. 10(c) and 10(d) our model was not able to exhibit this property. We can observe a memory effect if the extinction phase is not too long but the recall itself is 


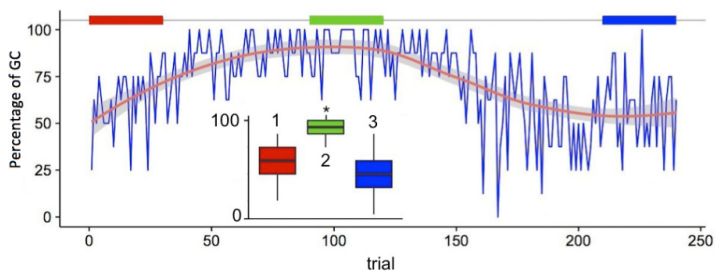

(a)

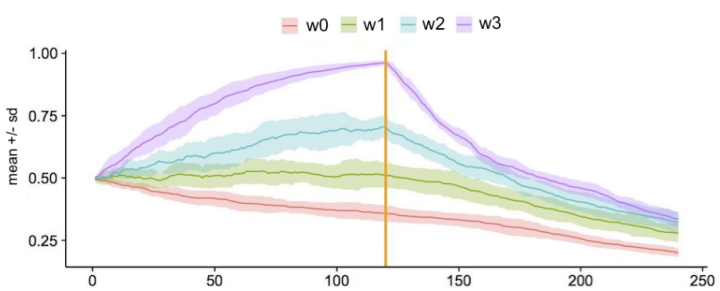

(b)

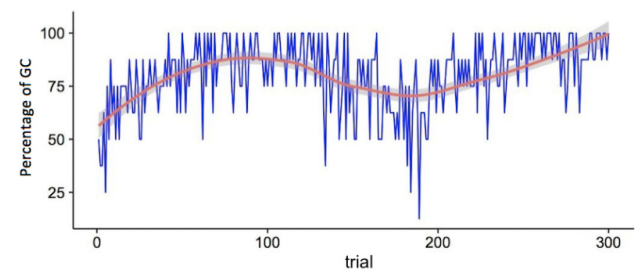

(c)

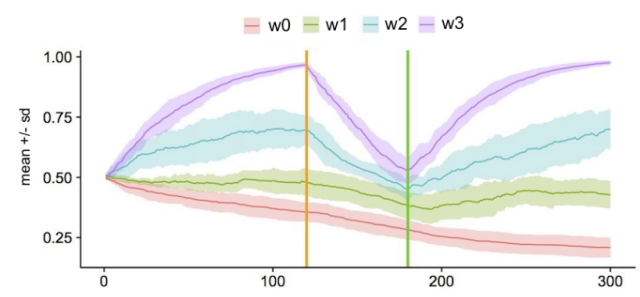

(d)

Figure 10: (Color online) Extinction protocol simulation results (a) Normalized rates of $G C$ averaged on 16 sessions during the single extinction protocol (red: smoothed curve obtained by a local polynomial regression fitting combined with a 95\% confidence interval). (A-inset) comparison of GC scores averaged on 30 trials samples (color boxes) recorded (1) at the beginning, (2) before extinction and (3) after extinction $(N=16 ; p<0: 05$, Kruskal-Nemenyi multiple comparison test). (b) variation of average cortico-striatal cognitive synaptic weights over time. $w_{i}$ is the average weight value for each of the four cues. The extinction starts at 120 trials (orange marker), (c) Normalized rates of $G C$ averaged on 16 sessions during a single extinction protocol followed by a recall. (d) variation of average cortico-striatal cognitive synaptic weights over time. The extinction starts at 120 trials (orange marker) and ends at 180 trials (recall: green marker).

not occurring faster. 


\section{Discussion}

\subsection{Following the flow of information}

We have demonstrated that a large-scale, neuron level model of BG circuitry could be implemented with reasonable computation power and was capable of making action selections in complex behavioral tasks. At this point of the discussion, the question usually comes up: why using a spike-based approach instead of a rate-based approach? The question remains open to decide whether or not, population rate-models can reliably mimic the true dynamics emerging of neural units interactions. This formalism is undeniably helpful when one tries to link large neuronal assemblies to behavioral observations. Yet, if we accept as true that the behavior of a system emerges from the interactions of its fundamental processing units, the model has sooner or later to be downscaled to this level to provide a more faithful and reliable description. This point has been very often discussed in the past and is still a matter of debate [Brette, 2015] but it seems that individual or relative spike-timing plays a major role in neural information encoding in different ways [Portelli et al., 2016, Jacobs et al., 2009, Saal et al., 2015, Moyer et al., 2014]. Models relying on individual spiking neurons and of which construction is constrained by anatomical and physiological evidence of complex connectivity architectures often provide fruitful results because they directly link a detailed circuitry to its assumed function or behavior [Chersi et al., 2013, Mandali et al., 2015, Medina et al., 2001, Yang et al., 2015]. 


\subsection{Comparison of action selection mechanisms}

Action selection requires that, of multiple alternatives presented, only one is chosen. This requires the use of a winner-takes-all architecture which usually relies on two different mechanisms. The first mechanism (i) relies on the feedback properties of the network itself. Our model is based on the presence of two feedback pathways having opposite effects and passing from cortex through the BG and back to cortex. On the basis of previous studies [Mink, 1996, Leblois et al., 2006a, Guthrie et al., 2013] we implemented a schematic BG action selection model in which, separate cortical networks of individual neurons are activated for each of a set of possible actions. In the model, the hyperdirect pathway negative feedback imposes its global inhibitory effect on cortical subregions. In parallel, the direct pathway exerts a more localized disinhibition which is gradually amplified and leads to the selection of an action. The second type of mechanism (ii) involves LI. This property has been observed in the striatum where domains of mutually inhibitory connections have been reported [Oorschot, 1996]. Many computational models have also explicitly used LI to produce "winner takes all" action selection networks involving competition mechanisms [Bar-Gad and Bergman, 2001, Suri and Schultz, 1999, Alexander and Wickens, 1993, Groves, 1983, Kotter and Wickens, 1998, Woodward et al., 1995, Wickens and Arbuthnott, 1993]. Even if recurrent inhibition is often considered as weak compared to feedforward inhibition [Jaeger et al., 1994, Koos et al., 2004, Tunstall et al., 2002], it has recently been emphasized that its role had to be considered [Moyer et al., 2014] and our simulations have shown its positive effect on learning capabilities of the BG. 


\subsection{Two-level action selection}

Previous models of BG [Gurney et al., 2001, Leblois et al., 2006b] have implemented selection between two choices at one level. In the current study, the presented cue is separated into two types of sensory stimulus (levels): the cue shape and its position [cf. Fig. 3(b)]. Under this interpretation, the action selection then requires two choices, one of the cue shape (cognitive component) and the other of the direction (motor component) which are implemented in parallel. This two-level decision making process was initially foreseen in a previous experimental study [Pasquereau et al., 2007]. A first implementation relying on population rate-models and mathematical transfer function was then developed in Guthrie et al., (2013) where anatomical evidences for its underlying architecture were also presented. In this study, the divergence in the cortical activations of cognitive and motor subpopulations was described as an emergent property of the network. The present model still performs the previously observed two-level action selection process and its related learning properties but, including additional circuitry elements and using a more detailed formalism, it extends this capability to new protocols and situations.

\subsection{Learning in the network}

In the naïve state, the network is able to spontaneously perform action selection. $\mathrm{SB}$ results from its connectivity pattern and intrinsic noise. Since no initial prewiring is present for any of the four-cue (or two-cue) protocol, GC and GD rates are close to $50 \%$ [Figs. $7(\mathrm{a})$ and $7(\mathrm{~b})$ ). The reinforcement learning process which takes place during a training session results from the synaptic weight 
changes of the cognitive cortex projections toward striatum and is modulated by the phasic variations in dopamine concentrations which have been shown to be necessary both for the LTP and for the LTD [Pawlak and Kerr, 2008, Kerr and Wickens, 2001, Reynolds et al., 2001]. Our model predicts efficiently the experimental observations of the four-cue and two-cue tasks [Pasquereau et al., 2007, Piron et al., 2016].

Its learning properties also exhibit consistent dynamics when submitted to reversal learning and extinction protocols. However, it also shows its limitation on these occasions. Indeed, during the reversal learning protocol, it fails to switch quicker to a new configuration as it is experimentally reported [Costa et al., 2015]. Similarly, during the extinction protocol, it does not exhibit a faster recall. These limitations do not necessarily throw doubt over the consistency of the model. They just highlight the fact that other important regions are not explicitly included in the model like the hippocampus which is involved in reversal learning [Shohamy et al., 2009] and the amygdala which is also known to play an important role in extinction process [Lingawi and Balleine, 2012].

\section{Conclusion}

We have presented here, for the first time, a biophysically based, spiking neuron model of the BG that is able to perform action selection explicitly relying on the interaction between the cognitive and motor levels. This model is closely based on the known anatomy and physiology of the BG and illustrates a reasonable 
network mechanism of decision making. In addition, it confirms that the action selection can be driven by noise, but that simulated learning can overcome this noise to produce optimum action selection performances [Guthrie et al., 2013]. Our model bridges the gap between top-down mesoscopic approaches and bottom-up models relying on emerging properties of neuronal networks dynamics. It is also able to predict some important behavioral characteristics like localized lesion effect on learning, reversal learning and extinction protocol.

\section{Acknowledgments}

The authors want to thank Eman KHEDR (Neurology Department, Faculty of Medicine, Assiut University, Assiut, EGYPT) and the NEUROMED project, funded by the Capacities Programme in the European Union's 7th Framework Programme (FP7-REGPOT-245807).

Martin Guthrie and André Garenne contributed equally to this paper.

\section{References}

[Albin et al., 1989] Albin, R. L., Penney, J. B., and Young, A. B. (1989). The functional anatomy of basal ganglia disorders. Trends in Neuroscience, 12 (10):366-375. 3, 8

[Alexander et al., 1986] Alexander, G. E., Long, M. R. D., and Strick, P. L. (1986). Parallel organization of functionally segregated circuits linking basal ganglia and cortex. Annual Review of Neuroscience, 9:357-381. 3 
[Alexander and Wickens, 1993] Alexander, M. E. and Wickens, J. R. (1993). Analysis of striatal dynamics: The existence of two modes of behaviour. Journal of Theoretical Biology, 163:413-438. 31

[Bar-Gad and Bergman, 2001] Bar-Gad, I. and Bergman, H. (2001). Stepping out of the box: information processing in the neural networks of the basal ganglia. Current Opinion in Neurobiology, 11 (6):689-695. 3, 31

[Bar-Gad et al., 2003] Bar-Gad, I., Morris, G., and Bergman, H. (2003). Information processing, dimensionality reduction and reinforcement learning in the basal ganglia. Progress in Neurobiology, 71 (6):439-473. 3, 9

[Barker et al., 2014] Barker, J. M., Taylor, J. R., and Chandler, L. J. (2014). A unifying model of the role of the infralimbic cortex in extinction and habits. Learn. Mem., 21(9):441-448. 27

[Berthet et al., 2012] Berthet, P., Hellgren-Kotaleski, J., and Lansner, A. (2012). Action selection performance of a reconfigurable basal ganglia inspired model with hebbian-bayesian go-nogo connectivity. Frontiers in Behavioral Neuroscience, 6(65):10-3389. 4

[Berthet et al., 2016] Berthet, P., Lindahl, M., Tully, P. J., Hellgren-Kotaleski, J., and Lansner, A. (2016). Functional relevance of different basal ganglia pathways investigated in a spiking model with reward dependent plasticity. Front Neural Circuits, 10:53. 8

[Bevan et al., 1997] Bevan, M. D., Eaton, S. A., and Bolam, J. P. (1997). Synaptic targets of physiologically, neurochemically and morphologically 
characterized neurons of the rate globus pallidus. In Society of Neuroscience Abstract, volume 23, page 196. 8

[Bouton, 2004] Bouton, M. E. (2004). Context and behavioral processes in extinction. Learn. Mem., 11(5):485-494. 28

[Brette, 2015] Brette, R. (2015). Philosophy of the spike: Rate-based vs. spike-based theories of the brain. Front Syst Neurosci, 9:151. 30

[Brown et al., 1998] Brown, L. L., Smith, D. M., and Goldbloom, L. M. (1998). Organizing principles of cortical integration in the rat neostriatum: corticostriate map of the body surface is an ordered lattice of curved laminae and radial points. Journal of Computational Neurology, 392(4):468-488. 8

[Chang et al., 1981] Chang, H. T., Wilson, C. J., and Kitai, S. T. (1981). Single neostriatal efferent axons in the globus pallidus: a light and electron microscopic study. Science, 313:915-918. 8

[Chen et al., 2010] Chen, H., Zhuang, P., Miao, S.-H., Yuan, G., Zhang, Y.-Q., and Li, J.-Y. (2010). Neuronal firing in the ventrolateral thalamus of patients with parkinson's disease differs from that with essential tremor. Chinese Medical Journal, 123 (6):695-701. 15

[Chersi et al., 2013] Chersi, F., Mirolli, M., Pezzulo, G., and Baldassarre, G. (2013). A spiking neuron model of the cortico-basal ganglia circuits for goal-directed and habitual action learning. Neural Netw, 41:212-224. 30

[Cools et al., 2002] Cools, R., Clark, L., Owen, A. M., and Robbins, T. W. (2002). Defining the neural mechanisms of probabilistic reversal learning 
using event-related functional magnetic resonance imaging. J. Neurosci, 22(11):4563-4567. 26

[Cools et al., 2009] Cools, R., Frank, M. J., Gibbs, S. E., Miyakawa, A., Jagust, W., and D'Esposito, M. (2009). Striatal dopamine predicts outcome-specific reversal learning and its sensitivity to dopaminergic drug administration. $J$. Neurosci., 29(5):1538-1543. 14

[Costa et al., 2015] Costa, V. D., Tran, V. L., Turchi, J., and Averbeck, B. B. (2015). Reversal learning and dopamine: a bayesian perspective. J. Neurosci., 35(6):2407-2416. 26, 33

[Dombrovski et al., 2015] Dombrovski, A. Y., Szanto, K., Clark, L., Aizenstein, H. J., Chase, H. W., Reynolds, C. F., and Siegle, G. J. (2015). Corticostriatothalamic reward prediction error signals and executive control in late-life depression. Psychol Med, 45(7):1413-1424. 26

[Frank et al., 2001] Frank, M. J., Loughry, B., and O'Reilly, R. C. (2001). Interactions between frontal cortex and basal ganglia in working memory: A computational model. Cognitive, Affective and Behavioral Neuroscience, 1(2):137-160. 4

[Gerfen, 1985] Gerfen, C. R. (1985). Compartmental organization of projections from the striatum to the substantia nigra in the rat. Journal of Computational Neurology, 236(4):454-476. 8

[Ghodratitoostani et al., 2016] Ghodratitoostani, I., Zana, Y., Delbem, A. C., 
Sani, S. S., Ekhtiari, H., and Sanchez, T. G. (2016). Theoretical tinnitus framework: A neurofunctional model. Frontiers in Neuroscience, 10:370. 8

[Goodman and Packard, 2015] Goodman, J. and Packard, M. G. (2015). The memory system engaged during acquisition determines the effectiveness of different extinction protocols. Frontiers in Behavioral Neuroscience, 9:314. 27

[Groves, 1983] Groves, P. M. (1983). A theory of the functional organization of the neostriatum and the neostriatal control of voluntary movement. Brain research reviews, 286:109-132. 31

[Gurney et al., 2001] Gurney, K., Prescott, T. J., and Redgrave, P. (2001). A computational model of action selection in the basal ganglia. i. a new functional anatomy. Biological Cybernetics, 84(6):401-410. 3, 32

[Guthrie et al., 2013] Guthrie, M., Leblois, A., Garenne, A., and Boraud, T. (2013). Interaction between cognitive and motor cortico-basal ganglia loops during decision making: A computational study. Journal of Neurophysiology, 109(12):3025-3040. 5, 7, 9, 10, 24, 31, 34

[Hines et al., 2008] Hines, M. L., Markram, H., and Schurmann, F. (2008). Fully implicit parallel simulation of single neurons. Journal of computational neuroscience, 25:439-448. 5

[Humphries et al., 2006] Humphries, M. D., Stewart, R. D., and Gurney, K. N. (2006). A physiologically plausible model of action selection and oscillatory 
activity in the basal ganglia. The Journal of Neuroscience, 26(50):1292112942. 9

[Hunter, 2007] Hunter, J. D. (2007). Matplotlib: A 2d graphics environment. Computing in Science and Engineering, 9:90-95. 13

[Jacobs et al., 2009] Jacobs, A. L., Fridman, G., Douglas, R. M., Alam, N. M., Latham, P. E., Prusky, G. T., and Nirenberg, S. (2009). Ruling out and ruling in neural codes. Proc. Natl. Acad. Sci. U.S.A., 106(14):5936-5941. 30

[Jaeger et al., 1994] Jaeger, D., Kita, H., and Wilson, C. J. (1994). Surround inhibition among projection neurons is weak or nonexistent in the rat neostriatum. Journal of Neurophysiology, 72(5):2555-2558. 31

[Kerr and Wickens, 2001] Kerr, J. N. D. and Wickens, J. R. (2001). Dopamine $\mathrm{d}-1 / \mathrm{d}-5$ receptor activation is required for long-term potentiation in the rat neostriatum in vitro. Journal of Neurophysiology, 85:117-124. 14, 33

[Kita and Kita, 1994] Kita, H. and Kita, S. T. (1994). The morphology of globus pallidus projection neurons in the rat : an intracellular staining study. Brain Research, 636(2):308-319. 8

[Klopf, 1988] Klopf, H. (1988). Neural models of plasticity. In (ed). John H. Byrne et William O. Berry. 15

[Koos et al., 2004] Koos, T., Tepper, J., and Wilson, C. J. (2004). Comaparison of ipscs evoked by spiny and fast-spiking neurons in the neostriatum. Journal of Neuroscience, 24(36):7916-7922. 31 
[Kotter and Wickens, 1998] Kotter, R. and Wickens, J. R. (1998). Striatal mechanisms in parkinson's disease: new insights from computer modeling. Artificial Intelligence in Medicine, 13:37-55. 31

[Kropotov and Etlinger, 1999] Kropotov, J. D. and Etlinger, S. C. (1999). Selection of actions in the basal ganglia-thalamocortical circuits: Review and model. International Journal of Psychophysiology, 31:197-217. 3

[Leblois et al., 2006a] Leblois, A., Boraud, T., Meissner, W., Bergman, H., and Hansel, D. (2006a). Competition between feedback loops underlies normal and pathological dynamics in the basal ganglia. Journal of Neuroscience, 26(13):3567-3583. 4, 5, 9, 31

[Leblois et al., 2006b] Leblois, A., Meissner, W., Bezard, E., Bioulac, B., Gross, C. E., and Boraud, T. (2006b). Temporal and spatial alterations in gpi neuronal encoding might contribute to slow down movement in parkinsonian monkeys. The European Journal of Neuroscience, 24 (4):1201-1208. 32

[Lee et al., 2012] Lee, D., Seo, H., and Jung, M. W. (2012). Neural basis of reinforcement learning and decision making. Annual Review of Neuroscience, 35:287-308. 3

[Levy, 1997] Levy, R. (1997). Re-evaluation of the functional anatomy of the basal ganglia in normal and parkinsonian states. Neuroscience, 76:335-343. 8

[Lingawi and Balleine, 2012] Lingawi, N. W. and Balleine, B. W. (2012). Amyg- 
dala central nucleus interacts with dorsolateral striatum to regulate the acquisition of habits. J. Neurosci., 32(3):1073-1081. 33

[Mandali et al., 2015] Mandali, A., Rengaswamy, M., Chakravarthy, V. S., and Moustafa, A. A. (2015). A spiking Basal Ganglia model of synchrony, exploration and decision making. Front Neurosci, 9:191. 30

[Medina et al., 2001] Medina, J. F., Garcia, K. S., and Mauk, M. D. (2001). A mechanism for savings in the cerebellum. J. Neurosci., 21(11):4081-4089. 30

[Migliore et al., 2006] Migliore, M., Cannia, C., Lytton, W. W., Markram, H., and Hines, M. L. (2006). Parallel networks simulations with neuron. Journal of Computational Neuroscience, 21(2):119-129. 5

[Mink, 1996] Mink, J. W. (1996). The basal ganglia: focused selection and inhibition of competing motor programs. Progress in Neurobiology, 50:381425. $3,5,8,31$

[Morita and Kawaguchi, 2015] Morita, K. and Kawaguchi, Y. (2015). Computing reward-prediction error: an integrated account of cortical timing and basal-ganglia pathways for appetitive and aversive learning. Eur. J. Neurosci., 42(4):2003-2021. 26

[Moyer et al., 2014] Moyer, J. T., Halterman, B. L., Finkel, L. H., and Wolf, J. A. (2014). Lateral and feedforward inhibition suppress asynchronous activity in a large, biophysically-detailed computational model of the striatal network. Front Comput Neurosci, 8:152. 8, 9, 24, 30, 31 
[Nambu, 2011] Nambu, A. (2011). Somatotopic organization of the primate basal ganglia. Frontiers in Neuroanatomy, 5:26. 5

[Neymotin et al., 2011] Neymotin, S. A., Lee, H., Park, E., Fenton, A. A., and Lytton, W. W. (2011). Emergence of physiological oscillation frequencies in a computer model of neocortex. Frontiers in Systems Neuroscience, 5:19. 13

[Oorschot, 1996] Oorschot, D. E. (1996). Total number of neurons in the neostriatal, pallidal, subthalamic, and substantia nigral nuclei of the rat basal ganglia: A stereological study using the cavalieri and optical disector methods. Journal of Comparative Neurology, 366 (4):580-599. 31

[Papachristou et al., 2013] Papachristou, H., Nederkoorn, C., Beunen, S., and Jansen, A. (2013). Dissection of appetitive conditioning. does impulsivity play a role? Appetite, 69:46-53. 27

[Pasquereau et al., 2007] Pasquereau, B., Nadjar, A., Arkadir, D., Bezard, E., Goillandeau, M., Bioulac, B., Gross, C. E., and Boraud, T. (2007). Shaping of motor responses by incentive values through the basal ganglia. Journal of Neuroscience, 27(5):1176-1183. 5, 6, 15, 32, 33

[Pawlak and Kerr, 2008] Pawlak, V. and Kerr, J. N. D. (2008). Dopamine receptor activation is required for corticostriatal spike-timing dependent plasticity. Journal of Neuroscience, 28(10):2435-2446. 14, 33

[Percheron et al., 1984] Percheron, G., Yelnik, J., and François, C. A. (1984). A golgi analysis of the primate globus pallidus. iii. spatial organization of the 
striato-pallidal complex. Journal of Comparative Neurology, 227(2):214-227. 7

[Piron et al., 2016] Piron, C., Kase, D., Topalidou, M., Goillandeau, M., Orignac, H., N'Guyen, T.-H., Rougier, N., and Boraud, T. (2016). The globus pallidus pars interna in goal-oriented and routine behaviors: Resolving a long-standing paradox. Movements Disorders, 31(8):1146-1154. 5, 6, 24,33

[Portelli et al., 2016] Portelli, G., Barrett, J. M., Hilgen, G., Masquelier, T., Maccione, A., Di Marco, S., Berdondini, L., Kornprobst, P., and Sernagor, E. (2016). Rank order coding: a retinal information decoding strategy revealed by large-scale multielectrode array retinal recordings. eNeuro, 3(3):18. 30

[R-Team, 2015] R-Team, editor (2015). R: A Language and Environment for Statistical Computing v. 3.2.2, R Foundation for Statistical Computing. 13

[Redgrave et al., 1999] Redgrave, P., Prescott, T. J., and Gurney, K. (1999). The basal ganglia: a vertebrate solution to the selection problem? Neuroscience, 89(4):1009-1023. 3

[Reynolds et al., 2001] Reynolds, J., Hyland, B., and Wickens, J. R. (2001). A cellular mechanism of reward-related learning. Nature, 413:67-70. 14, 33

[Saal et al., 2015] Saal, H. P., Harvey, M. A., and Bensmaia, S. J. (2015). Rate and timing of cortical responses driven by separate sensory channels. Elife, 4:e10450. 30 
[Sandoz and Pham-Delegue, 2004] Sandoz, J. C. and Pham-Delegue, M. H. (2004). Spontaneous recovery after extinction of the conditioned proboscis extension response in the honeybee. Learn. Mem., 11:586-597. 28

[Sarvestani et al., 2013] Sarvestani, I. K., Kozlov, A., Harischandra, N., Grillner, S., and Örjan Ekeberg (2013). A computational model of visually guided locomotion in lamprey. Biological Cybernetics, 107 (5):497-512. 4

[Schaette and Kempter, 2012] Schaette, R. and Kempter, R. (2012). Computational models of neurophysiological correlates of tinnitus. Front Syst Neurosci, 6:34. 8

[Schroll et al., 2014] Schroll, H., Vitay, J., and Hamker, F. H. (2014). Dysfunctional and compensatory synaptic plasticity in parkinson's disease. Eur. J. Neurosci., 39(4):688-702. 9

[Shohamy et al., 2009] Shohamy, D., Myers, C. E., Hopkins, R. O., Sage, J., and Gluck, M. A. (2009). Distinct hippocampal and basal ganglia contributions to probabilistic learning and reversal. J Cogn Neurosci, 21(9):1821-1833. 33

[Smith and Bolam, 1989] Smith, Y. and Bolam, J. P. (1989). Neurons of the substantia nigra reticulata receive a dense gaba-containing input from the globus pallidus in the rat. Brain Research, 493:160-167. 8

[Sukumar et al., 2012] Sukumar, D., Rengaswamy, M., and Chakravarthy, S. V. (2012). Modeling the contributions of basal ganglia and hippocampus to spatial navigation using reinforcement learning. PLoS One, 7(10):e47467. 4 
[Suri and Schultz, 1999] Suri, R. E. and Schultz, W. (1999). A neural network model with dopamine-like reinforcement signal that learns a spatial delayed response task. Neuroscience, 91 (3,):871-890. 3, 31

[Tunstall et al., 2002] Tunstall, M., Oorschot, D. E., Kean, A., and Wickens, J. R. (2002). Inhibitory interactions between spiny projection neurons in the rat striatum. Journal of Neurophysiology, 88(3):1263-1269. 31

[Wickens and Arbuthnott, 1993] Wickens, J. R. and Arbuthnott, G. W. (1993). The corticostriatal system on computer simulation: an intermediate mechanism for sequencing of actions. Prog. Brain Res., 99:325-339. 31

[Woodward et al., 1995] Woodward, D. J., Kirillov, A. B., Myre, C. D., and Sawyer, S. F. (1995). Neostriatal circuitry as a scalar memory: Modeling and ensemble neuron recording. In Houk, J. C., Davis, J. L., and Beiser, D. G., editors, Models of Information Processing in the Basal Ganglia, pages 315-336. The MIT Press, Cambridge, MA. 31

[Xue et al., 2013] Xue, G., Xue, F., Droutman, V., Lu, Z. L., Bechara, A., and Read, S. (2013). Common neural mechanisms underlying reversal learning by reward and punishment. PLoS ONE, 8(12):e82169. 26

[Yang et al., 2015] Yang, S., Wang, J., Li, S., Deng, B., Wei, X., Yu, H., and Li, H. (2015). Cost-efficient FPGA implementation of basal ganglia and their Parkinsonian analysis. Neural Netw, 71:62-75. 30 